\title{
Identification of miRNAs associated with sexual maturity in chicken ovary by Illumina small RNA deep sequencing
}

\author{
Li Kang ${ }^{1}$, Xinxing Cui ${ }^{1}$, Yujie Zhang ${ }^{1,2}$, Chunhong Yang ${ }^{1}$ and Yunliang Jiang ${ }^{1 *}$
}

\begin{abstract}
Background: MicroRNAs have been suggested to play important roles in the regulation of gene expression in various biological processes. To investigate the function of miRNAs in chicken ovarian development and folliculogenesis, two small RNA libraries constructed from sexually mature (162-day old) and immature (42-day old) ovary tissues of Single Comb White Leghorn chicken were sequenced using Illumina small RNA deep sequencing.
\end{abstract}

Results: In the present study, 14,545,100 and 14,774,864 clean reads were obtained from sexually mature (162-d) and sexually immature (42-d) ovaries, respectively. In total, 202 known miRNAs were identified, and 93 of them were found to be significantly differentially expressed: 42 miRNAs were up-regulated and 51 miRNAs were down-regulated in the mature ovary compared to the immature ovary. Among the up-regulated miRNAs, gga-miR-1a has the largest fold-change (6.405-fold), while gga-miR-375 has the largest fold-change (11.345-fold) among the down-regulated miRNAs. The three most abundant miRNAs in the chicken ovary are gga-miR-10a, gga-let-7 and gga-miR-21. Five differentially expressed miRNAs (gga-miR-1a, 21, 26a, 137 and 375) were validated by real-time quantitative RT-PCR (qRT-PCR). Furthermore, the expression patterns of the five miRNAs were analyzed in different developmental stages of chicken ovary and follicles of various sizes.

Conclusion: The present study provides the first miRNA profile in sexually immature and mature chicken ovaries. Some miRNAs such as gga-miR-1a and gga-miR-21are expressed differentially in immature and mature chicken ovaries as well as among different sized follicles, suggesting an important role in the follicular growth or ovulation mechanism in the chicken.

\section{Background}

In chicken, ovarian function determines laying performance. It has been estimated that approximately 12,000 oocytes are present in the hen ovary when sexually mature, but only a few hundred oocytes are selected to reach maturity and ovulate. Usually, the functionally mature hen ovary contains hundreds of white cortical follicles with 1-5 mm in diameter, 5-6 small yellow pre-hierarchical follicles with 6-8 $\mathrm{mm}$ in diameter and 5-6 large yellow preovulatory hierarchy follicles with $9-40 \mathrm{~mm}$ in diameter [1]. In the hen ovary, a single follicle is selected into the preovulatory hierarchy from a small cohort of pre-hierarchical follicles approximately once a day [2]. The preovulatory follicles are

\footnotetext{
*Correspondence: yljiang723@aliyun.com

'Shandong Provincial Key Laboratory of Animal Biotechnology and Disease Control and Prevention, College of Animal Science and Veterinary Medicine, Shandong Agricultural University, Taian 271018 Shandong Province, P.R. China Full list of author information is available at the end of the article
}

arranged in a hierarchical order in which the largest one (F1) represents the most mature and will ovulate for egg formation first. Ovary development and folliculogenesis undergo a series of complex processes including modulation of gonadotropin action, steroid hormones biosynthesis, follicle selection, granulosa cell proliferation and differentiation, which require tightly regulated expression and interaction of a multitude of genes at the transcriptional and post-transcriptional levels [3-7].

MicroRNAs (miRNAs) are a class of endogenous noncoding small regulatory RNAs of 19-24 nucleotides in size $[8,9]$ that can regulate gene expression by targeting specific sites in the $3^{\prime}$-untranslated region ( $3^{\prime}$-UTR) of mRNAs [10-13]. The seed region, which is located at miRNA nucleotides 2-8 from the $5^{\prime}$ end of a miRNA sequence, is the most important sequence for interaction with mRNA targets $[14,15]$. Increasing evidence indicates that miRNAs play important roles in post-transcriptional

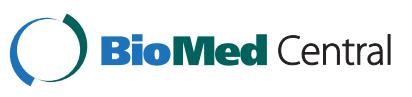


regulation of gene expression and have been shown to control multiple biological and metabolic processes such as organogenesis, development, cell proliferation and differentiation as well as many diseases [16-20]. The mechanism of miRNA regulated gene expression is very complex because one miRNA can target several thousand mRNAs, and one mRNA can be regulated by several miRNAs $[8,21]$. Identifying the expression patterns of miRNAs in the hen ovary is the first step to elucidating their functions in ovarian development and folliculogenesis. Several studies have demonstrated that inactivation of Dicer1 can result in accelerated early follicle recruitment and degeneration as well as infertility and the dysfunction of oocyte maturation and ovulation [22-24]. Furthermore, genome-wide miRNA expression has been examined in the ovaries of mice [25,26], cattle [27-29], pig [30] and sheep [31]. These studies show that miRNAs play a critical role in the development of mammalian gonads, but little is known about the involvement of miRNAs in adult chicken ovary and follicle development. The aim of this study was to identify the differentially expressed miRNAs in sexually mature and immature chicken ovaries by using Illumina small RNA deep sequencing (Illumina Genome Analyzer). This approach is highly suited for small RNA discovery, which not only provides sequences of low abundance species but also provides quantitative data as the frequency of sequencing reads reflects the abundance of miRNAs in the population [32]. Finally, we validated and identified the expression pattern of gga-miR-1a, 21, 26a, 137 and 375 at different developmental stages of ovary and follicles of various sizes using qRTPCR. These results may help us to further understand how ovarian function is regulated by miRNAs.

\section{Results}

\section{Sequence analysis of small RNAs in chicken ovary}

To survey miRNAs involved in the maturity of the chicken ovary, two small RNA libraries were generated from 42-d and 162-d chicken ovaries that, respectively, represent sexually immature and mature ovaries, and the libraries were sequenced by Illumina small RNA deep sequencing technology. In total, 16,817,204 and 17,936,768 raw reads were obtained from the sexually mature (162-d) and sexually immature (42-d) ovary libraries, respectively. After filtering the low-quality sequences, empty adaptors and single-read sequences, $14,545,100$ and $14,774,864$ clean reads of 18-30 nt were selected for further analysis from the sexually mature (162-d) and sexually immature (42-d) ovary libraries, respectively. Among the selected reads, 9,619,834 sequences from the mature ovary and $11,019,895$ sequences from the immature ovary mapped perfectly to the chicken genome (Additional file 1: Table S1), amounting to 66.14 and $74.59 \%$ of the total reads, respectively; and 5,834,400 reads in mature and 3,919,973 reads in immature ovaries were found to be similar to
miRNAs. The rest of the sequences were found to be other types of RNA, including noncoding RNA, tRNA, rRNA, snRNA or snoRNA. The number and proportions of the categories of small RNAs found are given in Additional file 1: Table S1.

The size distribution of small RNAs (sRNAs) was similar in the two libraries, and the majority of them were from 20 to 24 nt (Figure 1). The most abundant size class was $22 \mathrm{nt}$, which accounted for $36.12 \%$ and $23.58 \%$ of the total reads in sexually mature and immature chicken ovaries, respectively, followed by 23 nt (18.39\%, $14.54 \%)$ and 21 nt $(9.39 \%, 10.2 \%)$.

\section{Identification of known miRNAs in chicken ovary}

To investigate the expression of known miRNAs in the chicken ovary, small RNA sequences identified by Illumina small RNA deep sequencing were compared with known mature miRNAs and precursors in miRBase (version 14, 2009 and version 19, 2012). The results showed that 232 mature miRNA, 35 miRNA*, 260 miRNA-5p and 3396 miRNA-3p were found in the two libraries, and 202 known miRNAs are co-expressed in the two libraries (Additional file 1: Table S2).

The Illumina small RNA deep sequencing approach allows us to determine the relative abundance of various miRNA families by calculating the sequencing frequency. A highly expressed miRNA will likely have a large number of sequenced clones [33]. In the mature and immature chicken ovary libraries, known miRNAs had a broad range of expression levels; some (such as miR-10a, 21 and 101) were found to have more than hundreds of thousands of sequence reads, while others (such as miR-124a, 3540 and 1759) had less than 20 (Additional file 1: Table S2), indicating that expression varies significantly among different miRNA families. The proportion of different categories of small RNAs often reflects the roles in a particular tissue or different developmental stages and their associated biological mechanisms. The following 15 miRNAs were dominantly expressed in the two libraries: gga-miR-10a, gga-miR146c, gga-miR-101, gga-miR-21, gga-let-7a, gga-let-7b, ggalet-7c, gga-let-7j, gga-let-7f, gga-let-7 k, gga-miR-30a-5p, gga-miR-30e, gga-miR-148a, gga-miR-100 and gga-miR-126. Each of these 15 miRNAs had more than 100,000 reads, while the majority of other miRNAs sequenced had more than 500 reads. The most abundant miRNA in the mature ovary was gga-miR-10a, which had 1,177,256 reads, followed by gga-miR-21, which had 929,545 reads. However, in the immature ovary, gga-let-7a (415,122 reads) and gga-let-7j (413,833 reads) were the most abundant (Additional file 1: Table S2). Sequence analysis indicated that the relative abundance of members within one miRNA family varied greatly in the chicken ovary, suggesting functional divergence within the family. For example, in the immature ovary, ggalet-7 family abundance varied from 855 reads (gga-let-7d) to 


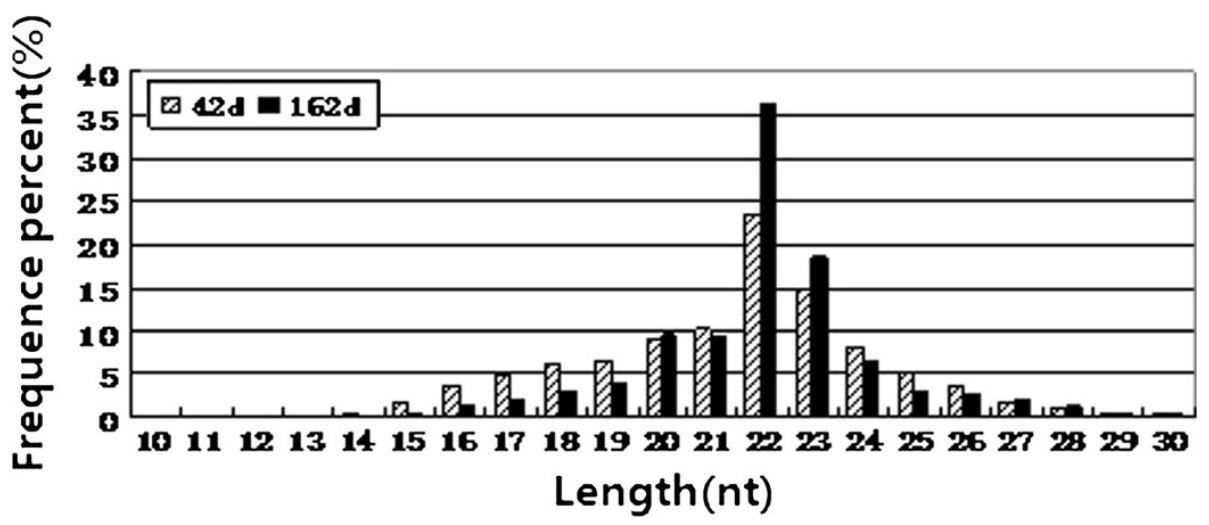

Figure 1 Length distribution and abundance of small RNAs sequences in chicken ovary by Illumina small RNA deep sequencing. Sequence length distribution of clean reads based on the abundance and distinct sequences; the most abundant size class was $22 \mathrm{nt}$, followed by $23 \mathrm{nt}$ and $21 \mathrm{nt}$.

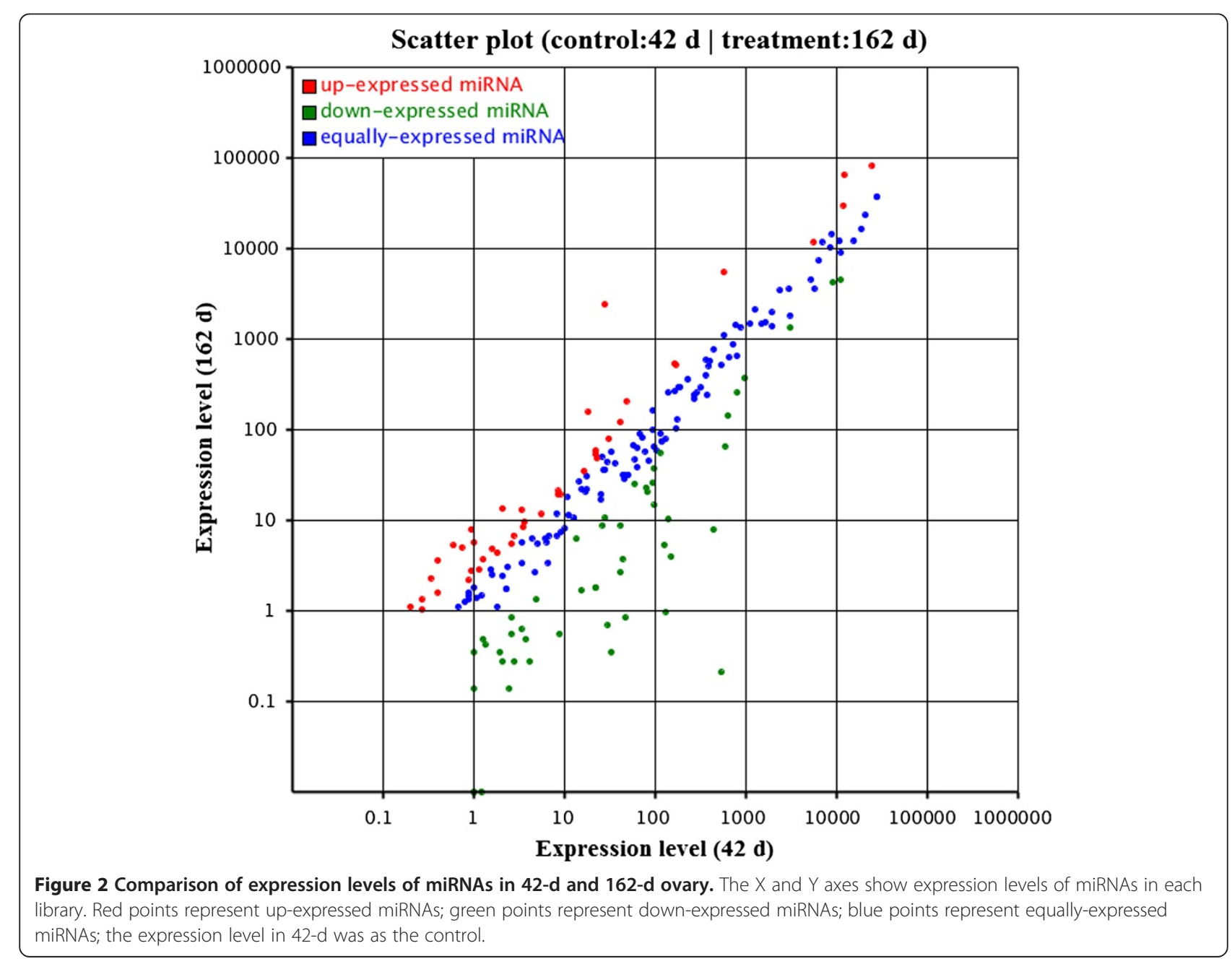


415,122 reads (gga-let-7a). Similarly, other miRNA families exhibited vast read ranges, such as the gga-miR146 (91235,844 reads) and gga-miR-30 (886-164,361 reads) families (Additional file 1: Table S2). These results indicate that different members within one miRNA family can have clearly different expression levels, most likely due to tissue- or developmental stage-specific expression.

In the two developmental stages of the chicken ovary, a total of 19 miRNA*s were co-expressed (Additional file 1: Table S3). It has been demonstrated that the miRNA* is generally less stable than their mature miRNA counterparts. During the biogenesis of the mature miRNAs, some of the miRNA* strands are degraded rapidly [34,35]. Generally, the miRNA* has lower expression levels than their corresponding mature miRNAs. In our data, the majority of miRNA*s had much fewer reads than their corresponding mature miRNAs. One intriguing exception was gga-miR $-140 \%$, which was present as 105,474 reads in the mature ovary and 95,992 reads in the immature ovary; however, gga-miR-140 had only 7,370 and 7,953 reads in each library, respectively. Similar expression patterns were found for gga-miR-199* and gga-miR-202* (Additional file 1: Table S3). Another interesting case was gga-miR-551*, in which only miRNA* was detected. These results suggest that miRNA*s may play some functional roles during chicken ovary development.

\section{miRNAs differentially expressed in sexually mature and immature chicken ovaries}

The main purpose of the study was to identify miRNAs involved in chicken ovary and follicle development. According to the changes in relative miRNA abundance between the two libraries, 93 miRNAs were expressed significantly differently between sexually mature and immature ovaries (Additional file 1: Table S4). In comparison with the sexually immature ovary (42-d), 42 miRNAs were significantly up-regulated, and 51 miRNAs were significantly down-regulated in the sexually mature ovary $(162-\mathrm{d}) \quad(\mid \log 2$ Ratio $\mid \geq 1$, $\mathrm{P}$-value $\leq 0.05$ ) (Additional file 1: Table S4). The majority of differentially expressed miRNAs range from a 2 - to 4-fold difference, and only 15 miRNAs showed differences greater than 4-fold between the two libraries (Figure 2 and Table 1). Among the up-regulated miRNAs, gga-miR-1a had the highest fold-change with 6.405-fold. Among the down-regulated miRNAs, ggamiR-375 had the highest fold-change with 11.345-fold, followed by gga-miR-217, gga-miR-458b, gga-miR449c and gga-miR-124a with more than 6-fold (Additional file 1: Table S4). In addition, we found that two members of the let-7 miRNA family, gga-let-7b and gga-let$7 \mathrm{~g}$, exhibited significantly differential expression, although the fold-changes were lower at 1.292- and 1.366-fold, respectively.
To validate the Illumina small RNA deep sequencing data, five differentially expressed miRNAs (gga-miR-1a, gga-miR-21, gga-miR-26a, gga-miR-137 and gga-miR-375) were selected, and their expression levels were quantified using real-time quantitative RT-PCR (qRT-PCR). The results were consistent with the deep sequencing data (Figure 3). These results provide evidence that Illumina small RNA deep sequencing is a sensitive and reliable approach to identifying differentially expressed miRNAs in the chicken ovary.

\section{Developmental expression patterns of five miRNAs in the ovary and follicles}

To further characterize the functionality of these differentially expressed miRNAs identified from the chicken ovary, the expression levels of gga-miR-1a, gga-miR-21, gga-miR-26a, gga-miR-137 and gga-miR-375 were further examined in ovary tissues from 42-, 70-, 90-, 110- and 162-day-old White Leghorn hens $(\mathrm{n}=3)$, as well as in follicles isolated from ovaries of 162-day-old White Leghorn hens, namely, a large white follicle (LW, diameter $=2$ $4 \mathrm{~mm}$ ), small yellow follicle (SF, diameter $=6-8 \mathrm{~mm}$ ), F6 (diameter $=12-14 \mathrm{~mm}$ ), F4 (diameter $=22-24 \mathrm{~mm}$ ), F2 (diameter $=30-31 \mathrm{~mm}$ ) and F1 (diameter $=34 \mathrm{~mm}$ ) follicles.

The results showed that some of the miRNAs exhibit developmental stage-specific expression patterns in ovarian development (Figure 4). The expression patterns of gga-miR-1a and gga-miR-21 were similar in the different developmental stages; relatively lower expression was observed from 42-d to 110-d compared with 162-d and

\begin{tabular}{|c|c|c|c|c|}
\hline miR-name & Fold-change & P-value & regulated & Sig-lable \\
\hline gga-miR-375 & 11.345 & 0 & down & $* *$ \\
\hline gga-miR-217 & 7.1082 & 0 & down & ** \\
\hline gga-miR-458b-5p & 6.9287 & $4.42 \mathrm{E}-06$ & down & $* *$ \\
\hline gga-miR-449c-5p & 6.6656 & $3.46 \mathrm{E}-05$ & down & $* *$ \\
\hline gga-miR-124a & 6.6656 & $3.46 \mathrm{E}-05$ & down & $* *$ \\
\hline gga-miR-216a & 6.5919 & $1.08 \mathrm{E}-136$ & down & $* *$ \\
\hline gga-miR-10a & 6.4055 & 0 & up & $* *$ \\
\hline gga-miR-34b & 5.8478 & $8.82 \mathrm{E}-188$ & down & $* *$ \\
\hline gga-miR-7 & 5.8327 & 0 & down & $* *$ \\
\hline gga-miR-216b & 5.4757 & $3.08 \mathrm{E}-188$ & down & $* *$ \\
\hline gga-miR-34c & 5.2853 & 0 & down & $* *$ \\
\hline gga-miR-137 & 4.5848 & 0 & down & $* *$ \\
\hline gga-miR-1720-3p & 4.1869 & $1.98 \mathrm{E}-09$ & down & $* *$ \\
\hline gga-miR-9-3p & 4.0218 & $6.41 \mathrm{E}-30$ & down & $* *$ \\
\hline gga-miR-135a & 4.0032 & 1.33E-134 & down & $* *$ \\
\hline
\end{tabular}

** represents fold change $(\log 2)>1$ or fold change $(\log 2)<-1$, and $P$-value $<0.01$. 

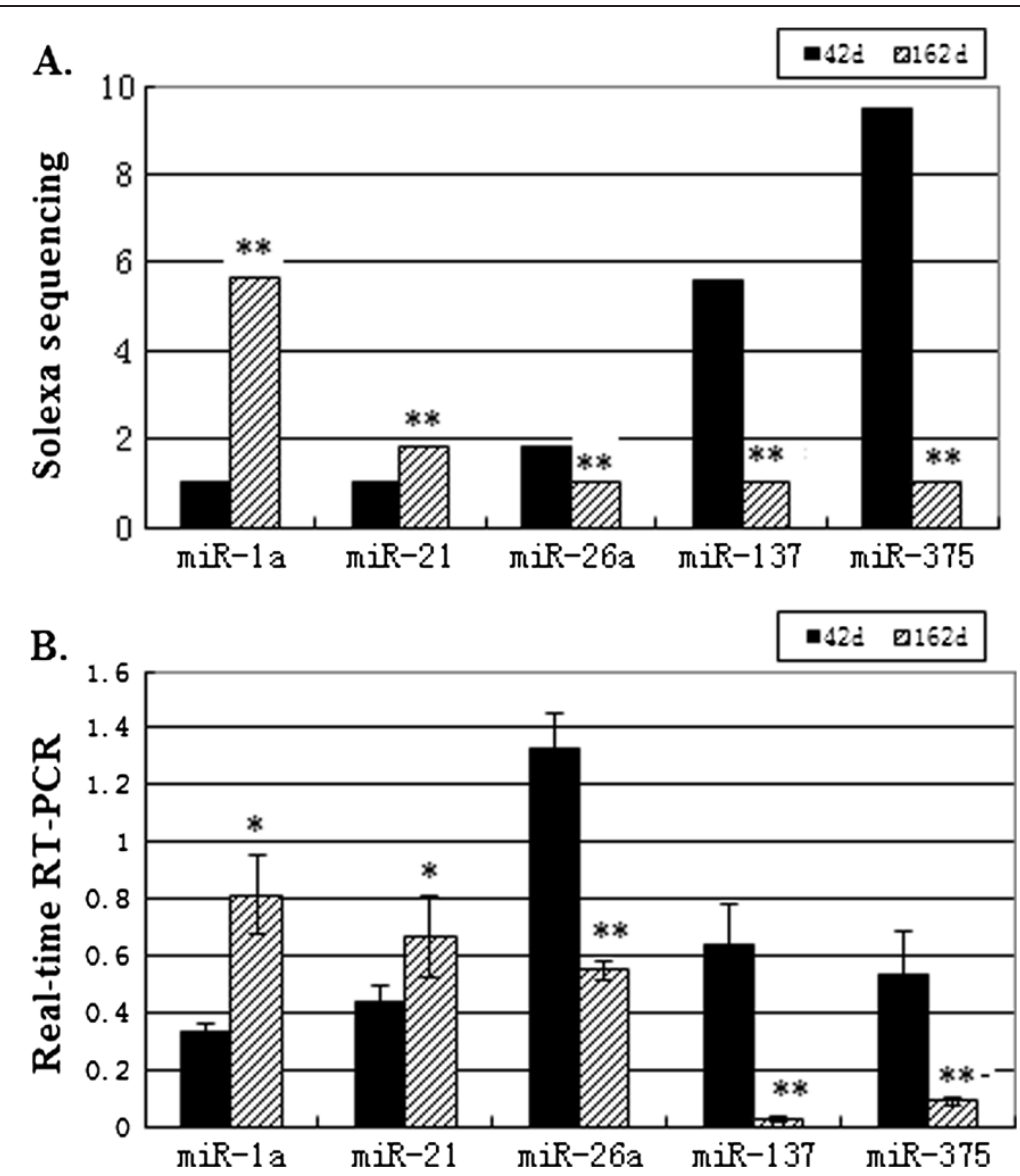

Figure 3 qRT-PCR validation of five differentially expressed miRNAs identified using Illumina small RNA deep sequencing. $A$. Foldchange of five miRNAs that were differentially expressed between $42-d$ and 162-d ovaries based on deep sequencing data. $\boldsymbol{B}$. The relative expression abundance of the five miRNAs between $42-d$ and 162 - $d$ ovaries by real-time quantitative RT-PCR. ${ }^{*} P<0.05$, ${ }^{* *} P<0.01$.

increased dramatically to peak in 162-d. However, the expression dynamics of gga-miR-26a were different; the highest expression level was found in the 42-d ovary, then decreased from 70-d to $110-\mathrm{d}$, and finally increased in the 162-d ovary although still lower than $42-\mathrm{d}$. The expression of gga-miR-137 and gga-miR-375 in ovary decreased significantly from 42 -d to 162 -d.

In follicles (Figure 4), the expression patterns of ggamiR-1a and gga-miR-21 were similar; during the development from LW to F1, the expression levels of the two miRNAs increased progressively with $\mathrm{F} 1$ having the highest level, which suggests that gga-miR-1a and ggamiR-21 may be involved in the follicular growth or ovulation mechanism in the chicken. For gga-miR-26a and gga-miR-137, the highest expression level was found in the SF $(6-8 \mathrm{~mm})$ follicle. The expression of gga-miR-375 was relatively lower in LW and SF follicles, increased most in the F6-F2 follicles and then declined in the F1 stage, suggesting an important role of gga-miR-26a and gga-miR-137 in hierarchy maintenance of follicles in chicken.

\section{Target prediction and function annotation}

To further understand the physiological functions and biology processes involved by 93 differentially expressed miRNAs during ovary development, target prediction was performed by integrating three public databases (TargetScan, PicTar and miRanda), and 3,122 target genes were identified (data not shown). GO annotation and KEGG pathway analysis were performed to identify functional modules regulated by these miRNAs. The GO annotation enrichment results showed that regulation of biological process, cellular metabolic process, developmental process and signal transduction are the most significantly enriched GO terms (Figure 5). The KEGG pathway analysis revealed 178 pathways that were enriched with miRNA targets (data not shown). MAPK signaling pathway, Calcium signaling pathway, Wnt-signaling pathway Insulin signaling pathway and $\mathrm{GnRH}$ signaling pathway ranked among the most enriched pathways (Table 2). Although the false-positive predictions always exist, we suggest that these targets have high possibility of being regulated by miRNAs in chicken ovary development. 


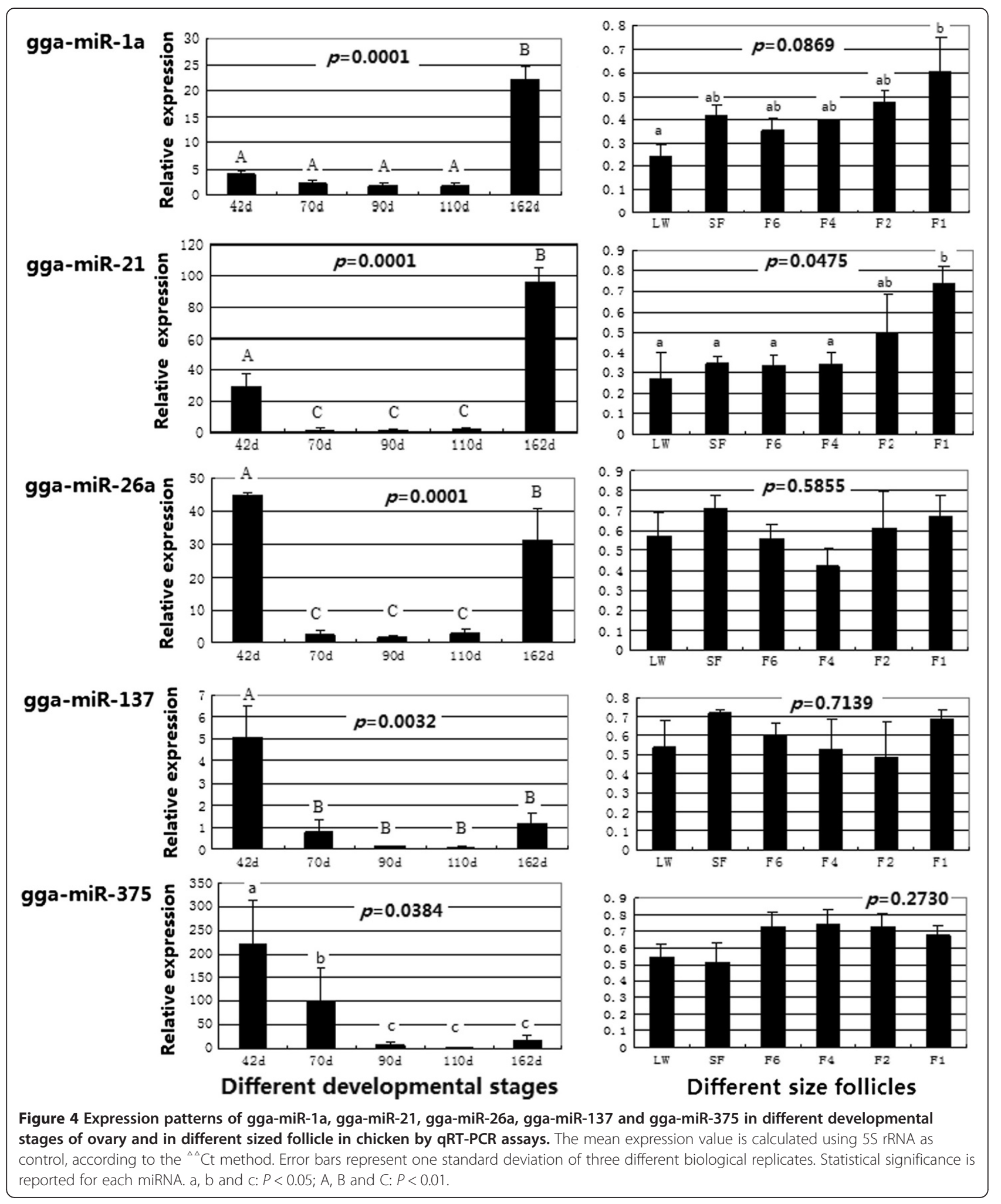

\section{Discussion}

The Illumina deep sequencing platform is efficient for miRNA discovery and is widely used to generate small
RNA profiles in various organisms. In the present study, we obtained detailed miRNA profiles of sexually mature (162-d) and immature (42-d) chicken ovaries using this 


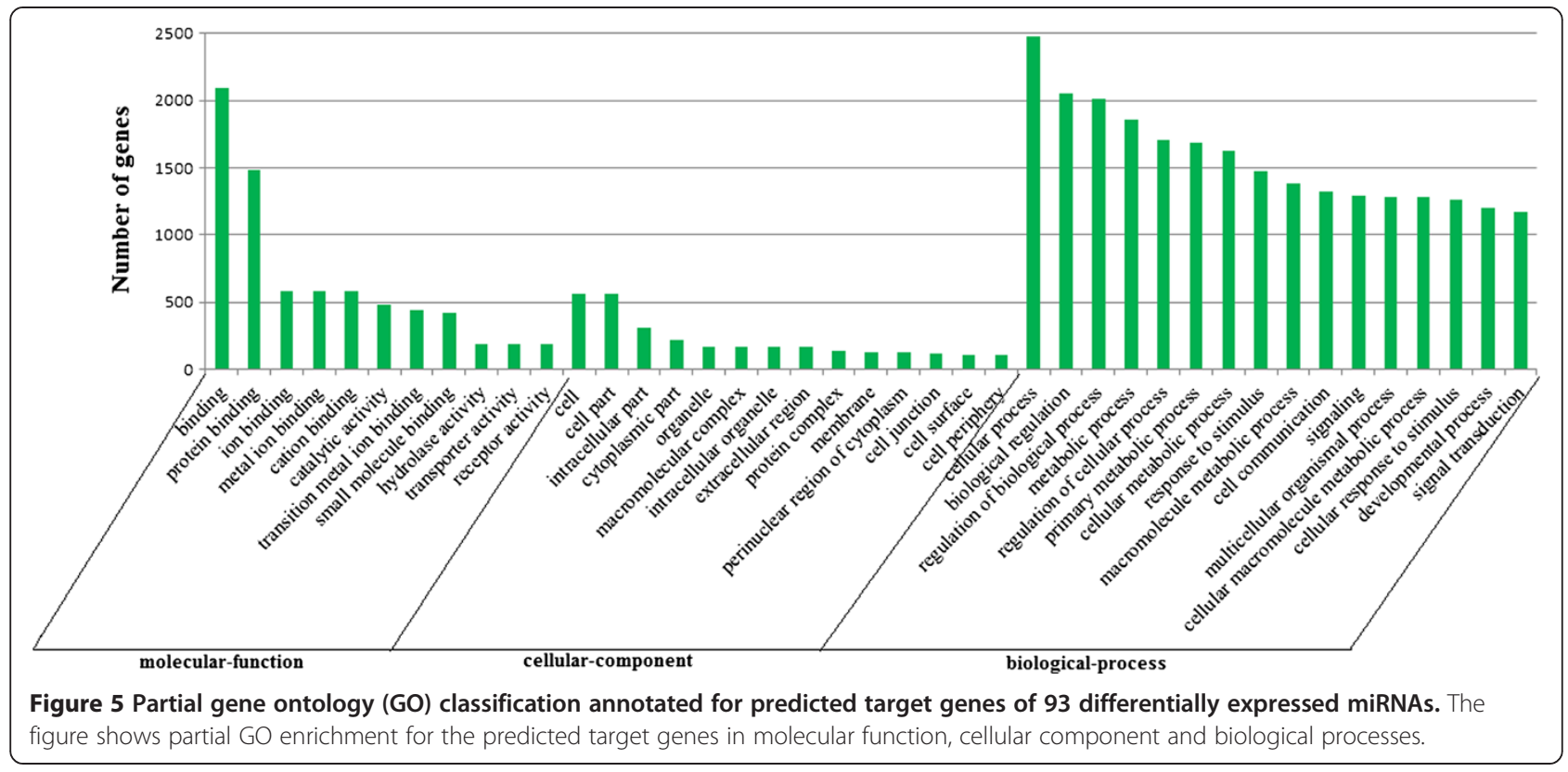

method. To our knowledge, this is the first report of miRNA expression profiles of sexually immature and mature ovaries in the chicken. The sequence analysis showed that the dominant size of small RNAs in chicken ovary was $22 \mathrm{nt}$ followed by 23 and $21 \mathrm{nt}$ sequences. This result is typical of Dicer-processed small RNA products and was consistent with the known 19-24 nt range for miRNAs. Our data are consistent with previous findings in mouse [25] and pig [30] testis and ovary as well as chicken skeletal muscle [36] and somites [37]. However, in Holstein Cattle testis and ovary, the $20 \mathrm{nt}$

Table 2 The most enriched KEGG pathways of target genes for differentially expressed miRNAs

\begin{tabular}{llll}
\hline Pathway & $\begin{array}{l}\text { Genes } \\
\text { count }\end{array}$ & $\begin{array}{l}\text { P- } \\
\text { value }\end{array}$ & $\begin{array}{l}\text { Pathway } \\
\text { ID }\end{array}$ \\
\hline MAPK signaling pathway & 320 & 0.8154 & ko04010 \\
Calcium signaling pathway & 264 & 0.8454 & ko04020 \\
ECM-receptor interaction & 210 & 0.9928 & ko04512 \\
Axon guidance & 210 & 0.8752 & ko04360 \\
Wnt signaling pathway & 209 & 0.8757 & ko04310 \\
Insulin signaling pathway & 191 & 0.8859 & ko04910 \\
Oocyte meiosis & 146 & 0.9117 & ko04114 \\
TGF-beta signaling pathway & 123 & 0.9251 & ko04350 \\
Gap junction & 122 & 0.9257 & ko04540 \\
GnRH signaling pathway & 121 & 0.9263 & ko04912 \\
PPAR signaling pathway & 108 & 0.9340 & ko03320 \\
Progesterone-mediated oocyte & 108 & 0.9340 & ko04914 \\
maturation & & & \\
p53 signaling pathway & 97 & 0.9405 & ko04115 \\
VEGF signaling pathway & 86 & 0.9471 & ko04370 \\
\hline
\end{tabular}

size is the most abundant, followed by 22 nt [29], while another study in bovine ovary indicated that $21 \mathrm{nt}$ is the predominant size [27]. This is likely caused by difference in species or developmental stages.

In the sexually mature chicken ovary library, ggamiR-10a and gga-miR-21 were the two most frequently sequenced miRNAs, and the let-7 miRNA family was another abundant cluster with let-7a being the most abundantly expressed miRNA. The let-7 miRNA family was also expressed abundantly in ovary and oocyte of bovines $[28,29,38,39]$, as well as in murine ovaries and testis [40]. Furthermore, gga-miR-101, gga-miR-1a, gga-miR-146c, gga-miR-148a, gga-miR-126, gga-miR26a and gga-miR-30d were abundant in our sequencing libraries, as has been shown in other animal gonads $[25,27,28]$. These miRNAs may have important roles in female reproductive physiology. A previous study reported that miR-21 was significantly up-regulated in murine granulosa cells before and $4 \mathrm{~h}$ after the hCG/ LH surge and that it plays a role in preventing apoptosis in periovulatory granulosa cells as they transit to luteal cells. Knockdown of miR-21 in granulosa cells resulted in increased apoptosis and was associated with a reduced ovulation rate $[41,42]$. Some studies have shown that miR-21 was down-regulated by estradiol in MCF7 cancer cells $[43,44]$. In the present study, ggamiR-21 exhibited a significant 2.39 -fold increase in a sexually mature ovary compared with an immature ovary, and the highest level was in F1 follicles, implying that gga-miR-21 plays an important role in sexual maturation in hens.

In this study, gga-let-7b is up-regulated while gga-let-7 g, gga-miR-17-3p gga-miR-30d, gga-miR-29a, gga-miR-26a 
and gga-miR-181a are all down-regulated in the mature ovary compared with the immature ovary. It has also been reported that miR-30c, let-7a, let-7b and let-7c were upregulated, while miR-30d, miR-29a and miR-26a were down-regulated in rat granulosa cells treated with FSH for $12 \mathrm{~h}$, which suggests that these miRNAs participate in FSH-mediated progesterone biosynthesis of granulosa cells [45]. Another study showed that a lack of miR17-5p and let-7b resulted in corpus luteum insufficiency and infertility in mice and that exogenous administration of the two miRNAs could prevent this phenotype [46]. A recent study showed that the activated estrogen receptor can suppress the expression of BAX by up-regulating a group of miRNAs including hsa-let-7 family members in endometrial adenocarcinoma and precancerous lesions [47]. In the same study, miR-181a and miR-30d are significantly downregulated after estradiol treatment.

miR-26a is reported to have anti-apoptotic effects in many cancers [48-51]. In MCF7 cancer cells, estradiol can repress miR-26a expression to regulate numerous genes associated with cell growth and proliferation [44]. miR-26a was also found to play a role in normal tissue growth and development and to have an impact on cell proliferation and differentiation. One study showed that, during myogenesis, overexpression of miR-26a targets the enhancer of Zeste homolog 2 (Ezh2), which normally suppresses skeletal muscle differentiation [52]. In osteogenesis, miR-26a was found to regulate osteoblast cell growth and differentiation in human adipose tissue-derived stem cells [53]. In this study, the highest level of gga-miR-26a was found in SF follicles. SF were to be chosen from pre-hierarchical follicles $(<8 \mathrm{~mm})$ to pre-ovulatory hierarchy follicles $(>10 \mathrm{~mm})$, therefore, gga-miR-26a is likely associated with the mechanism of recruitment of dominant follicle in chicken.

Studies indicated that gga-miR-31, gga-miR-101, ggamiR-202 and gga-miR-202* may be involved in regulating gonadal differentiation in embryonic chicken gonads [54-56]. Reduced miR-202* expression is correlated with reduced expression of the testis-associated genes DMRT1 and SOX9, and up-regulation of the ovary-associated genes FOXL2 and CYP19A1 (aromatase) [55]. FOXL2 is a critical factor required for ovarian growth and differentiation whose functions include regulating aromatase enzyme expression, inhibins and follistatin gene expression, and granulosa cell development $[57,58]$. In our study, gga-miR $-202 \%$ was down-regulated by 3.2 -fold and gga-miR-31 was down-regulated by 1.68 -fold in sexually mature ovaries compared with immature ovaries, suggesting they are also involved in the sexual maturity of ovary.

Bioinformatic analysis shows that miR-101 targets TGIF1 (TGFB-induced factor homeobox 1), ZEB2 (zinc finger Ebox binding homeobox 2) and BMPR1B, which participate in the regulation of TGF- $\beta$ signaling [56]. TGF- $\beta$ signaling is critical to folliculogenesis and oogenesis in mammalian ovaries [59]. The increase in female miR-101 expression in differentiating ovaries can ease repression of TGF- $\beta$ signaling [60]. In this study, gga-miR-101 was abundantly expression in chicken ovary and 123 putative target genes were involved in TGF- $\beta$ signaling pathway. Study has shown that overexpression of miR-375 suppressed glucoseinduced insulin secretion, and conversely, inhibition of endogenous miR-375 function enhanced insulin secretion [61]. In this study, gga-miR-375 is the largest downregulated by 11 -fold in the mature ovary and 191 putative target genes were involved in insulin signaling pathway.

\section{Conclusion}

The present study is the first to examine the miRNA expression profile of sexually mature and immature ovaries in chicken, and evaluate miRNA function during ovary development and folliculogenesis. We identified 93 known miRNAs that were differentially expressed in mature and immature chicken ovaries that could exert novel functions in the regulation of ovarian development and folliculogenesis. Some miRNAs such as gga-miR-1a and gga-miR-21are expressed differentially in immature and mature chicken ovaries as well as among different sized follicles, suggesting an important role in the follicular growth or ovulation mechanism in the chicken. Further investigation concerning the function of these miRNAs should facilitate our understanding of the regulatory roles of miRNAs in regulating chicken ovary and follicle growth. The role of individual miRNAs in chicken ovarian development and follicle growth requires further investigation.

\section{Methods}

\section{Collection of chicken ovary and follicle tissues}

A total of 30 one-day-old female Single Comb White Leghorn chickens (Gallus gallus domesticus) were obtained from Shanghai Poultry Breeding Co Ltd and were reared in the brooding temperature at $35^{\circ} \mathrm{C}(65 \% \mathrm{RH})$ for the first 5 days. The lighting program was $23 \mathrm{~h}$ light: $1 \mathrm{~h}$ darkness. The chickens were reared under natural temperature and light (latitude: $36^{\circ} 28^{\prime} \mathrm{N}$; longitude: 117²59'; June to October, 2009). All chickens received a starter diet with 19\% crude protein and $11.97 \mathrm{MJ} / \mathrm{kg}$ of metabolizable energy; after day 42 they received a grower diet with $16.5 \%$ crude protein and $11.97 \mathrm{MJ} / \mathrm{kg}$ of metabolizable energy. Feed and water were freely available during the rearing period.

Samples were collected when the chickens were 42, 70, 90, 110 and 162 days-old. At each stage, three chickens were randomly slaughtered and ovaries were collected, and the following follicles were collected from 162-d ovary: F1 (34 mm), F2 (30-31 mm), F4 (22-24 mm), F6 (12$14 \mathrm{~mm}$ ), small yellow follicle (SF, 6-8 $\mathrm{mm}$ ) and large white follicle (LW, 2-4 mm). Further, three whole ovaries from 42- and 162-day-old Single Comb White Leghorn hen were 
collected separately to prepare two pools representing sexually immature and sexually mature ovaries for constructing small RNA libraries. These tissues were frozen in liquid nitrogen for RNA isolation.

All animal experiments were approved by the Institutional Animal Care and Use Ethics Committee of Shandong Agricultural University and performed in accordance with the "Guidelines for Experimental Animals" of the Ministry of Science and Technology (Beijing, China).

\section{Small RNA library construction and Illumina small RNA deep sequencing}

Two small RNA libraries pooled from immature ovaries (42-d) and mature ovaries (162-d) were constructed. Total RNA was extracted using Trizol (Invitrogen) according to the manufacturer's protocol, and the quantity of RNA was examined by using an Agilent 2100 Bioanalyzer. From each sample, $20 \mu \mathrm{g}$ of total RNA was used for Solexa sequencing by an Illumina Genome Analyzer (Illumina, San Diego, CA, USA) at the Beijing Genomics Institute (BGI) (Shenzhen, Guangzhou, China). Briefly, the 16-30 nt fraction of total RNA was purified and enriched using denaturing polyacrylamide gel electrophoresis (PAGE). Then $3^{\prime}$ and $5^{\prime}$ RNA adapters were each ligated with T4 RNA ligase. Subsequently, the small RNAs ligated to adapters were subjected to RT-PCR amplification for 15 PCR cycles. The amplification products (70-90 bases in length, small RNA and adapters) were further purified on a $6 \%$ polyacrylamide TBE gel and used for sequencing analysis. Sequencing reads were extracted from the image files generated by the Illumina/Solexa 1G Genome Analyzer.

\section{Bioinformatic analysis of sequencing data}

After filtering adaptor sequences and removal of contaminated reads, the clean reads were processed for computational analysis. First, the clean reads were mapped to the UCSC chicken genome galGal3 using NCBI MegaBLAST and rRNA, tRNA, snRNA, scRNA and snoRNA were discarded from the small RNA sequences. Subsequently, the remaining sequences were analyzed by a BLAST search against the miRNA database, miRBase (version14.0 and 19.0) [62], to identify the conserved miRNAs in Gallus gallus. Only the perfectly matched sequences were considered to be conserved miRNAs. To analyze differential miRNA, miRNAs expression in each library (42-d and 162d chicken ovary) was normalized to obtain the expression of transcripts per million using the following formula: Normalized expression $=($ Actual miRNA sequencing reads count $/$ Total clean reads count) $\times 1,000,000$. If the normalized expression (NE) value of a given miRNA is zero, the expression value was modified to 0.01 . If the normalized expression of a given miRNA is less than 1 in both libraries, it was removed in future differential expression analyses. The fold-change and P-value were calculated from the normalized expression. When $\mid \log 2$ Ratio $\mid \geq 1$ and P-value $\leq$ 0.05 , it was be seen as differential expression [63].

\section{Quantitative real-time PCR of miRNAs}

To validate and characterize the differentially expressed miRNAs identified using high-throughput sequencing, five miRNAs were selected, and we analyzed their relative expression levels in ovaries at 42, 70, 90, 110 and 162 days of age as well as in different sized follicles. Real-time quantitative PCR was performed using Mx3000p SYBR $^{\circ}$ Green Real-time quantitative PCR Analyzer (Stratagene, USA). Briefly, $3 \mu \mathrm{g}$ miRNA was reverse transcribed to cDNA using One Step PrimeScript ${ }^{\circ}$ miRNA cDNA Synthesis Kit (Tiangen Biotech Co., China). The reverse transcriptase reaction consisted of $10 \mu \mathrm{L} 2 \times$ miRNA Reaction Buffer Mix, $2 \mu \mathrm{L} 0.1 \%$ BSA, $2 \mu \mathrm{L}$ miRNA PrimeScript ${ }^{\oplus}$ RT Enzyme Mix, $2 \mu \mathrm{L}$ total RNA $(2 \mu \mathrm{g} / \mu \mathrm{L})$ and RNase-free $\mathrm{dH}_{2} \mathrm{O}$ up to $20 \mu \mathrm{L}$. The RT-PCR program was $37^{\circ} \mathrm{C}$ for $60 \mathrm{~min}$ and $85^{\circ} \mathrm{C}$ for $5 \mathrm{sec}$. The cDNA products were stored at $-20^{\circ} \mathrm{C}$. Real-time quantitative PCR was performed with $\mathrm{SYBR}^{\oplus}$ Premix Ex Taq ${ }^{\text {"m }}$ II (Tiangen Biotech Co., China). The reaction solution was prepared on ice, and the components were $10 \mu \mathrm{L}$ SYBR $^{\circledR}$ Premix Ex Taq II $(2 \times), 0.8 \mu \mathrm{L}$ PCR Forward Primer $(10 \mu \mathrm{M}), 0.8 \mu \mathrm{L}$ Uni-miR qPCR Primer $(10 \mu \mathrm{M}), 0.4 \mu \mathrm{L}$ ROX Reference Dye II $(50 \times), 3 \mu \mathrm{L}$ cDNA, and $\mathrm{dH}_{2} \mathrm{O}$ up to $20 \mu \mathrm{L}$. The reaction mixtures were incubated in a 96-well plate at $95^{\circ} \mathrm{C}$ for $30 \mathrm{sec}$ followed by 40 cycles of $95^{\circ} \mathrm{C}$ for $5 \mathrm{sec}, 60^{\circ} \mathrm{C}$ for $30 \mathrm{sec}$ and $72^{\circ} \mathrm{C}$ for $30 \mathrm{sec}$. All reactions were run in triplicate. The primers for miRNAs have the same sequences as Gallus gallus miRNAs with an appropriate adjustment at their $5^{\prime}$ terminus. The relative expression quantification was calculated using the $2^{-\Delta \Delta C t}$ method after the threshold cycle $(C t)$ and was normalized with the $C t$ of chicken 5S rRNA. Data are from three individuals. The each miRNA expression level was presented as $2^{-\Delta \Delta \mathrm{Ct}}$ means \pm SE (standard error), and error bars indicate the standard error of $2^{-\Delta \Delta C t}$ mean values. One-way ANOVA and Duncan's Multiple Range test $(P<0.05)$ (SAS version 8.02, 2001) were used to examine the significance of differential expression level in each miRNA between different stages ovaries or different size follicles, and the difference was considered as significant when $P<0.05$.

\section{Additional file}

Additional file 1: Table S1. Summary of Illumina small RNA deep sequencing data for small RNAs in mature and immature chicken ovaries. Table S2. The expression patterns of known miRNAs in the two libraries of chicken ovary. Table S3. The expression patterns of miRNA*s and their corresponding miRNAs in chicken ovary. Table S4. The significantly differentially expressed known miRNAs in chicken ovary.

\section{Competing interests}

The authors declare that they have no competing interests. 


\section{Authors' contributions}

LK designed and performed the experiments and drafted the manuscript. XC carried out the GRT-PCR. YZ and CY carried out animal care and prepared samples. YJ conceived the study and the experimental design and helped draft the manuscript. All authors read and approved the final manuscript.

\section{Acknowledgements}

We thank Dr. Huaxiang Yan and Dr. Changsuo Yang for providing the White Leghorn chickens. This research was supported by the National Natural Science Foundation of China (30871777, 31272435), and the Agricultural Elite Breeds (Poultry) Project of Shandong Province (2011LZ15).

\section{Author details}

'Shandong Provincial Key Laboratory of Animal Biotechnology and Disease Control and Prevention, College of Animal Science and Veterinary Medicine Shandong Agricultural University, Taian 271018 Shandong Province, P.R. China. ${ }^{2}$ College of Life Science, Linyi University, Linyi 276005, China.

Received: 12 October 2012 Accepted: 20 May 2013 Published: 26 May 2013

\section{References}

1. Onagbesan O, Bruggeman V, Decuypere E: Intra-ovarian growth factors regulating ovarian function in avian species: a review. Anim Reprod Sci 2009, 111(2-4):121-140.

2. Johnson AL, Woods DC: Dynamics of avian ovarian follicle development Cellular mechanisms of granulosa cell differentiation. Gen Comp Endocrinol 2009, 163(1-2):12-17.

3. Fortune JE, Rivera GM, Evans AC, Turzillo AM: Differentiation of dominant versus subordinate follicles in cattle. Biol Reprod 2001, 65(3):648-654.

4. Woods DC, Johnson AL: Regulation of Follicle-Stimulating Hormone receptor mRNA in hen granulose cells relative to follicle selection. Biol Reprod 2005, 72(3):643-650.

5. Hillier SG: Gonadotropic control of ovarian follicular growth and development. Mol Cell Endocrinol 2001, 179(1-2):39-46.

6. Matzuk MM: Revelations of ovarion follicle biology from gene knockout mice. Mol Cell Endocrinol 2000, 163(1-2):61-66.

7. Pannetier M, Mandon-Pépin B, Copelli S, Fellous M: Molecular aspects of female and male gonadal development in mammals. Pediatr Endocrinol Rev 2004, 1(3):274-287.

8. Bartel DP: MicroRNAs: Genomics, Biogenesis, Mechanism, and Function Cell 2004, 116(2):281-297.

9. Carrington JC, Ambros V: Role of microRNAs in plant and animal development. Science 2003, 301(5631):336-338.

10. Lewis BP, Shih $\mathrm{H}$, Jones-Rhoades MW, Bartel DP, Burge CB: Prediction of mammalian microRNA targets. Cell 2003, 115:787-798

11. Krol J, Loedige I, Filipowicz W: The widespread regulation of microRNA biogenesis, function and decay. Nat Rev Genet 2010, 11(9):597-610.

12. Ambros $\mathrm{V}$, Chen $\mathrm{X}$ : The regulation of genes and genomes by small RNAs. Develoment 2007, 134(9):1635-1641.

13. Zhang $B H$, Wang QL, Pan XP: MicroRNAs and their regulatory roles in animals and plants. J Cell Physio/ 2007, 210(2):279-289.

14. Brennecke J, Stark A, Russell RB, Cohen SM: Principles of micro-RNA-target recognition. PLOS Biol 2005, 3(3):e85.

15. Lewis BP, Burge CB, Bartel DP: Conserved seed pairing, often flanked by adenosines, indicates that thousands of human genes are microRNA targets. Cell 2005, 120:15-20.

16. Ambros V: The functions of animal microRNAs. Nature 2004, 431:350-355.

17. Alvarez-Garcia I, Miska EA: MicroRNA functions in animal development and human disease. Development 2005, 132(21):4653-4662.

18. Kloosterman WP, Plasterk RHA: The diverse functions of MicroRNAs in animal development and disease. Dev Cell 2006, 11(4):441-450.

19. Bushati N, Cohen SM: MicroRNA functions. Annu Rev Cell Dev Biol 2007 23:175-205

20. Zhang BH, Pan XP, Cobb GP, Anderson AT: microRNAs as oncogenes and tumor suppressors. Dev Biol 2007, 302:1-12

21. Bartel DP: MicroRNAs: target recognition and regulatory functions. Cell 2009, 136:215-233.

22. Lei L, Jin S, Gonzalez G, Behringer RR, Woodruff TK: The regulatory role of Dicer in folliculogenesis in mice. Mol Cell Endocrinol 2010, 315(1-2):63-73.
23. Murchison EP, Stein P, Xuan Z, Pan H, Zhang MQ, Schultz RM, Hannon GJ: Critical roles for Dicer in the female germline. Genes Dev 2007, 21:682-693.

24. Hong X, Luense LJ, McGinnis LK, Nothnick WB, Christenson LK: Dicer1 is essential for female fertility and normal development of the female reproductive system. Endocrinology 2008, 149:6207-6212

25. Mishima T, Takizawa T, Luo SS, Ishibashi O, Kawahigashi Y, Mizuguchi Y, Ishikawa T, Mori M, Kanda T, Goto T, Takizawa T: MicroRNA (miRNA) cloning analysis reveals sex differences in miRNA expression profiles between adult mouse testis and ovary. Reproduction 2008, 136(6):811-822.

26. Ro S, Song R, Park C, Zheng H, Sanders K, Yan W: Cloning and expression profiling of small RNAs expressed in the mouse ovary. RNA 2007, 13:2366-2380.

27. Hossain MM, Ghanem N, Hoelker M, Rings F, Phatsara C, Tholen E, Schellander K, Tesfaye D: Identification and characterization of miRNAs expressed in the bovine ovary. BMC Genomics 2009, 10:443.

28. Tripurani SK, Xiao C, Salem M, Yao J: Cloning and analysis of fetal ovary microRNAs in cattle. Anim Reprod Sci 2010, 120:16-22

29. Huang J, Ju Z, Li Q, Hou Q, Wang C, Li J, Li R, Wang L, Sun T, Hang S, Gao Y, Hou M, Zhong J: Solexa Sequencing of Novel and Differentially Expressed MicroRNAs in Testicular and Ovarian Tissues in Holstein Cattle. Int J Biol Sci 2011, 7(7):1016-1026.

30. Li M, Liu Y, Wang T, J G, Luo Z, Chen H, Wang X, Chen L, Ma J, Mu Z, Jiang A, Zhu L, Lang Q, Zhou X, Wang J, Zeng W, Li N, Li K, Gao X, Li X: Repertoire of Porcine MicroRNAs in Adult Ovary and Testis by Deep Sequencing. Int J Biol Sci 2011, 7((7):1045-1055.

31. McBride D, Carre W, Sontakke S, Hogg CO, Law AS, Donadeu FX, Clinton M: Identification of miRNAs associated with the follicular luteal transition in the ruminant ovary. Reproduction 2012, 144(2):221-233.

32. Burnside J, Ouyang M, Anderson A, Bernberg E, Lu C, Meyers BC, Green PJ, Markis M, Isaacs G, Huang E, Morgan RW: Deep sequencing of chicken microRNAs. BMC Genomics 2008, 9:185.

33. van Ommen GJ, den Dunnen JT: Deep sequencing-based expression analysis shows major advances in robustness, resolution and inter-lab portability over five microarray platforms. Nucleic Acids Res 2008, 36:e141.

34. Okamura K, Ishizuka A, Siomi H, Siomi MC: Distinct roles for Argonaute proteins in small RNA-directed RNA cleavage pathways. Genes Dev 2004, 18:1655-1666.

35. Okamura K, Phillips MD, Tyler DM, Duan H, Chou YT, Lai EC: The regulatory activity of microRNA* species has substantial influence on microRNA and 3' UTR evolution. Nat Struct Mol Biol 2008, 15:354-363.

36. Li T, Wu R, Zhang Y, Zhu D: A systematic analysis of the skeletal muscle miRNA transcriptome of chicken varieties with divergent skeletal muscle growth identifies novel miRNAs and differentially expressed miRNAs. BMC Genomics 2011, 12:186.

37. Rathjen T, Pais H, Sweetman D, Moulton V, Munsterberg A, Dalmay T: High throughput sequencing of microRNAs in chicken somites. FEBS Lett 2009, 583:1422-1426.

38. Tesfaye D, Worku D, Rings F, Phatsara C, Tholen E, Schellander K, Hoelker M: Identification and expression profiling of microRNAs during bovine oocyte maturation using heterologous approach. Mol Reprod Dev 2009, 76:665-677.

39. Miles JR, McDaneld TG, Wiedmann RT, Cushman RA, Echternkamp SE, Valle $J$, Smith TPL: MicroRNA expression profile in bovine cumulus-oocyte complexes: Possible role of let-7 and miR-106a in the development of bovine oocytes. Anim Reprod Sci 2012, 130:16-26.

40. Reid JG, Nagaraja AK, Lynn FC, Drabek RB, Muzny DM, Shaw CA, MK W e, Naghavi AO, Khan M, Zhu H, Tennakoon J, Gunaratne GH, Corry DB, Miller J, McManus MT, German MS, Gibbs RA, Matzuk MM, Gunaaratne PH: Mouse let-7 miRNA populations exhibit RNA editing that is constrained in the 5'-seed/cleavage/anchor regions and stabilize predicted mmu-let-7a: mRNA duplexes. Genome Res 2008, 18:1571-1581.

41. Fiedler SD, Carletti MZ, Hong X, Christenson LK: Hormonal Regulation of MicroRNA Expression in Periovulatory Mouse Mural Granulosa Cells. Bio Reprod 2008, 79:1030-1037

42. Carletti MZ, Fiedler SD, Christenson LK: MicroRNA 21 Blocks Apoptosis in Mouse Periovulatory Granulosa Cells. Biol Reprod 2010, 83(2):286-295.

43. Wickramasinghe NS, Manavalan TI, Dougherty SM, Riggs KA, Li Y, Klinge CM: Estradiol downregulates miR-21 expression and increases miR-21 target gene expression in MCF-7 breast cancer cells. Nucleic Acids Res 2009, 37(8):2584-2595.

44. Maillot G, Lacroix-Triki M, Pierredon S, Gratadou L, Sabine S, Bénès V, Roché $H$, Dalenc F, Auboeuf D, Millevoi S, Vagner S: Widespread estrogendependent repression of microRNAs involved in breast tumor cell growth. Cancer Res 2009, 69(21):8332-8340. 
45. Yao N, Yang B-Q, Liu Y, Tan X-Y, Lu C-L, Yuan X-H, Ma X: Follicle stimulating hormone regulation of microRNA expression on progesterone production in cultured rat granulosa cells. Endocrine 2010 38:158-166.

46. Otsuka M, Zheng M, Hayashi M, Lee JD, Yoshino O, Lin S, Han J: Impaired microRNA processing causes corpus luteum insufficiency and infertility in mice. J Clin Invest 2008, 118:1944-1954.

47. Zhang R, He Y, Zhang X, Xing B, Sheng Y, Lu H, Wei Z: Estrogen receptorregulated microRNAs contribute to the $B C L 2 / B A X$ imbalance in endometrial adenocarcinoma and precancerous lesions. Cancer Lett 2012, 314(2):155-165.

48. Zhang B, Liu XX, Zhou CX, Guo M, He M, Li MF, Chen GQ, Zhao Q: Pathologically decreased miR-26a antagonizes apoptosis and facilitates carcinogenesis by targeting MTDH and EZH2 in breast cancer. Carcinogenesis 2011, 32(1):2-9.

49. Lu J, Getz G, Miska EA, Alvarez-Saavedra E, Lamb J, Peck D, Sweet-Cordero A, Ebert BL, Mak RH, Ferrando AA, Downing JR, Jacks T, Horvitz RH, Golub TR: MicroRNA expression profiles classify human Cancers. Nature 2005, 435:834-838.

50. Saito Y, Jones PA: Epigenetic activation of tumor suppressor microRNAs in human cancer cells. Cell Cycle 2006, 5(19):2220-2222.

51. Garzon R, Fabbri M, Cimmino A, Calin GA, Croce CM: MicroRNA expression and function in cancer. Trends Mol Med 2006, 12(12):580-587.

52. Wong CF, Tellam RL: MicroRNA-26a targets the histone methyltransferase Enhancer of Zeste homolog 2 during myogenesis. J Biol Chem 2008, 283(15):9836-9843.

53. Luzi E, Marini F, Sala SC, Tognarini I, Galli G, Brandi ML: Osteogenic differentiation of human adipose tissue-derived stem cells is modulated by the miR-26a targeting of the SMAD1 transcription factor. J Bone Miner Res 2008, 23(2):287-295.

54. Bannister SC, Tizard ML, Doran TJ, Sinclair AH, Smith CA: Sexually dimorphic microRNA expression during chicken embryonic gonadal development. Biol Reprod 2009, 81:165-176.

55. Bannister SC, Smith CA, Roeszler KN, Doran TJ, Sinclair AH, Tizard ML: Manipulation of estrogen synthesis alters MIR202* expression in embryonic chicken gonads. Biol Reprod 2011, 85(1):22-30.

56. Cutting AD, Bannister SC, Doran TJ, Sinclair AH, Tizard MVL, Smith CA: The potential role of microRNAs in regulating gonadal sex differentiation in the chicken embryo. Chromosome Res 2012, 20:201-213.

57. Blount AL, Schmidt K, Justice NJ, Vale WW, Fischer WH, Bilezikjian LM: Foxl2 and Smad3 coordinately regulate follistatin gene transcription. $J$ Biol Chem 2009, 284(12):7631-7645.

58. Schmidt D, Ovitt CE, Anlag K, Fehsenfeld S, Gredsted L, Treier AC, Treier M: The murine wingedhelix transcription factor Foxl2 is required for granulose cell differentiation and ovary maintenance. Development 2004, 131(4):933-942

59. Knight PG, Glister C: TGF-beta superfamily members and ovarian follicle development. Reproduction 2006, 132(2):191-206.

60. Vaillant S, Magre S, Dorizzi M, Pieau C, Richard-Mercier N: Expression of AMH, SF1, and SOX9 in gonads of genetic female chickens during sex reversal induced by an aromatase inhibitor. Dev Dyn 2001, 222(2):228-237.

61. Poy MN, Eliasson L, Krutzfeldt J, Kuwajima S, Ma X, MacDonald PE, Pfeffer S, Tuschl T, Rajewsky N, Rorsman P, Stoffel M: A pancreatic islet-specific microRNA regulates insulin secretion. Nature 2004, 432:226-230.

62. Kozomara A, Griffiths-Jones S: miRBase: integrating microRNA annotation and deep-sequencing data. Nucleic Acids Res 2011, 39(suppl 1):D152-D157.

63. Audic S, Claverie JM: The singnificance of digital gene expression profiles. Genome Res 1997, 7(10):986-995.

doi:10.1186/1471-2164-14-352

Cite this article as: Kang et al: Identification of miRNAs associated with sexual maturity in chicken ovary by lllumina small RNA deep sequencing. BMC Genomics 2013 14:352.

\section{Submit your next manuscript to BioMed Central and take full advantage of:}

- Convenient online submission

- Thorough peer review

- No space constraints or color figure charges

- Immediate publication on acceptance

- Inclusion in PubMed, CAS, Scopus and Google Scholar

- Research which is freely available for redistribution

Submit your manuscript at www.biomedcentral.com/submit
Biomed Central 